\title{
Numerical simulation of dynamic TPB fracture test in a modified Hopkinson bar
}

\author{
J.Loya, J. Fernandez-Saez and C. Navarro
}

Department of Continuum Mechanics and Structural Analysis, Carlos III University of Madrid, Avda. de la Universidad 30, 28911 Leganés, Madrid, Spain

\begin{abstract}
Dynamic three point bending fracture tests performed in a modified Hopkinson Bar are used to obtain material fracture properties (such as the fracture-initiation toughness) at high strain rates. This work presents a three-dimensional numerical analysis of the aforementioned tests, performed by the Finite Element Method, implemented in the commercial code ABAQUS. The relationship between the Stress Intensity Factor and the Crack Mouth Opening Displacement was examined and compared with the bidimensional one. The results indicate that the use of $2 \mathrm{D}$ plane strain solutions can underestimate the fracture properties.
\end{abstract}

\section{INTRODUCTION}

Integrity of mechanical and structural components subjected to dynamic loading requires to know the material fracture behaviour at high strain rates. Considering mode I, several dynamic fracture parameters may be defined in relation to the crack propagation regime: the dynamic fracture-initiation toughness, $K_{I d}$, represents the value of the Stress Intensity Factor, SIF, at which a crack starts to propagate. Other dynamic fracture parameters are $K_{I D}$ (dynamic fracture propagation toughness) and $K_{I A}$ (crack arrest toughness). These three material fracture parameters are used in design but the first is of special significance because it rates the effective propagation of a crack within the structural element subjected to impulsive load.

In contrast to the determination of static fracture toughness, $K_{I C}$, the methodology for dynamic fracture initiation toughness, $K_{I d}$, is not yet standardized. The instrumented Charpy test has been widely used to evaluate the dynamic fracture properties of materials, but the maximum loading rate (Stress Intensity Factor loading rate, $\dot{K}_{I}$ ) achieved during the test is about $\dot{K}_{I}=10^{5} \mathrm{MPa} \sqrt{\mathrm{m}} / \mathrm{s}$. Descriptions have been published $[1,2,3,4]$ of special arrangements of the Split Hopkinson Pressure $\operatorname{Bar}(S H P B)$ for dynamic bending tests at higher strain rates.

This Hopkinson tests permit higher strain rates than those reached by instrumented Charpy impact tests. The system consists of a striker bar (called projectile or impactor), an input pressure bar with a modified shape edge, a supporting device and the recording equipment. The cracked specimen is placed between the input bar and the supporting device and is loaded to fracture by means of a concentrated transverse force applied at its midspan. The projectile, moving at velocity $V_{0}$, strikes the input bar, generating a longitudinal strain compressive pulse, $\varepsilon_{i}(t)$, that propagates along the bar. This pulse can be recorded by strain gages on its outer surface. Once the pulse reaches the right edge of the bar, part of its energy is directly transmitted to the specimen and to the supporting device, while the remaining energy is reflected back to the input bar, but now, as a tensile pulse, $\varepsilon_{r}(t)$. The reflected pulse is recorded by the strain gages. Assuming the theory of onedimensional elastic wave propagation, the load exerted on the specimen, $P_{i}(t)$, and the displacement of the edge of the bar initially in contact with the specimen, $u(t)$, can be calculated $[1,2,3,4]$.

To obtain $K_{I d}$ experimentally, both the time history of the Stress Intensity Factor, $K_{I}(t)$, during the specimen loading process, and the instant, $t_{f}$, at which the crack in the material begins to grow must be determined. Then $K_{I d}$ can be defined as:

$$
K_{I d}=K_{I}\left(t_{f}\right)
$$

To evaluate the SIF throughout dynamic tests, different optical $[5,6,7]$ and photoelastic [8] 
techniques have been proposed, but in general they require complex equipment.

Numerical approaches [2], [9] based on the Finite Element Method (FEM) have been used to study the problem. Others $[10,11]$ derived simple formulas for the dynamic SIF of three-point bending specimens using the Euler-Bernouilli [10] or Timoshenko [11] beam theories, modelling the specimen as a simply supported beam with a crack at its midspan. These last models would be strictly applicable if pure three-point bending test conditions were maintained throughout the experiment, that is, with no contact loss between the specimen and the support device, nor between the specimen and the input bar. However, recent two-dimensional numerical analysis of the dynamic three point bending test [12], accounting for the possible loss of contact between the interacting bodies, showed that the dynamic $S I F$ can be evaluated with high precision from the measurement of Crack Mouth Opening Displacement $C M O D$ throughout the test, assuming that the same relationships between SIF and CMOD used in the static cases apply to the dynamic ones. In this last work [12] a high speed photography system was used to measure the $C M O D$ directly on the specimen. Popelar et al. [13] proposed a dynamic test method in which the $C M O D$ was measured by means of a gage.

The aforementioned analyses are based on 2-D solutions and less information about 3-D analyses is found in the literature, but several studies have considered three-dimensional effects in the determination of fracture properties [14, 15].

Here we present a three-dimensional numerical analysis of the dynamic three point bending fracture tests performed in a modified Hopkinson Bar. The numerical analysis is by Finite Element Method, implemented in the commercial code ABAQUS. The relationship between the SIF and the $C M O D$ is examined and compared with the bidimensional one.

\section{NUMERICAL SIMULATION}

A three-dimensional numerical simulation of the impact bending fracture test was performed by the Finite Element Method using the commercial code ABAQUS [16]. The finite element model includes the projectile, the input bar, the specimen and its supporting device. The simulated projectile and input bar were cylindrical, $22 \mathrm{~mm}$ in diameter and $330 \mathrm{~mm}$ and $1 \mathrm{~m}$ length, respectively. One of the input bar edges, precisely that in contact with the specimen, is sketched in figure 1.

The geometry and dimensions of the specimen are given in figure 2. Its dimensions were: width $W=20 \mathrm{~mm}$, span $S=80 \mathrm{~mm}$, and total length, $L=100 \mathrm{~mm}$. The specimen had a fatigue crack of length $a$. Three dimensional effects were studied varying the thickness, $B,(5 \mathrm{~mm}$ and $10 \mathrm{~mm})$ and the crack length $(a / W=0.3,0.5$ and 0.7$)$.
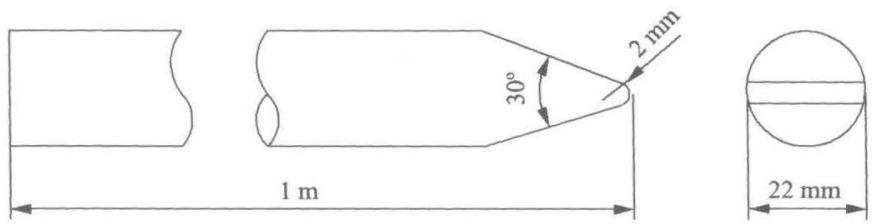

Figure 1: Input bar scheme.

Given the symmetry in geometry and boundary conditions, only one quarter of the input bar, projectile and specimen, and only half of one roller support were modelled. The input bar mesh was of 3020 nodes and 1988 elements, and that of the projectile had 870 nodes and 550 elements.

For the mesh of the specimen, eight layers were used through the thickness. To take into account the stress and strain concentrations at the crack tip, the mesh was refined in this zone, the smallest element side being of $2.8 \mu \mathrm{m}$. The mesh was also refined at the contact zone between the specimen and the input bar, and between the specimen and the supporting device.

The number of nodes and elements used in the mesh of the specimen depends on the case analyzed, and varies between 5982 and 6174 nodes, and 7303 and 7744 elements.

The supporting device was modelled by a half-roller $8 \mathrm{~mm}$ in diameter. To simulate the rest of the support, 60 special elements called "infinity elements" (CIN3D8) provided by ABAQUS finite element package [16] were added. This kind of element is often used in boundary value problems 

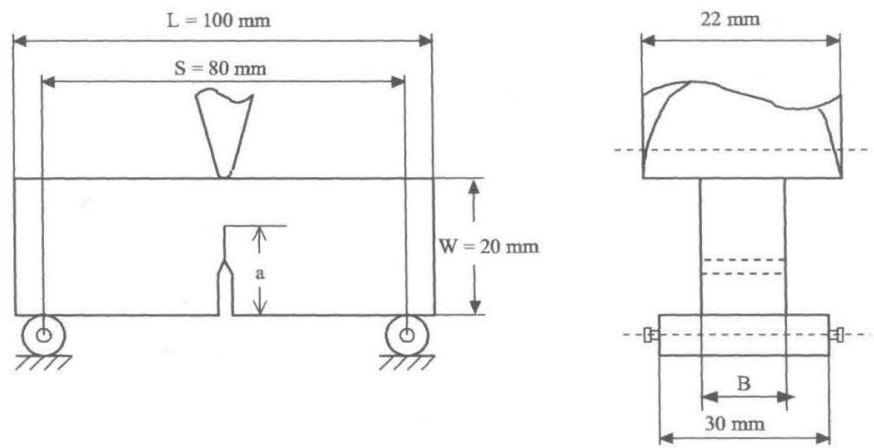

Figure 2: Bending specimen scheme.

defined in unbounded domains, or for problems in which the region of interest is small as compared to the surrounding medium. These last elements can be used in conjunction with conventional finite elements [16]. In this dynamic analysis, the infinity elements were chosen for their ability to transmit energy outside the finite element mesh, without trapping or reflecting it. This transmission is optimized when the boundary between finite and infinite elements is orthogonal to the direction from which the waves impinge on this boundary [16]. Figure 3 shows the meshes for the specimen, the end of the input bar and the support used in the analysis for the case of $B=10 \mathrm{~mm}$ and $\mathrm{a} / W$ $=0.5$.
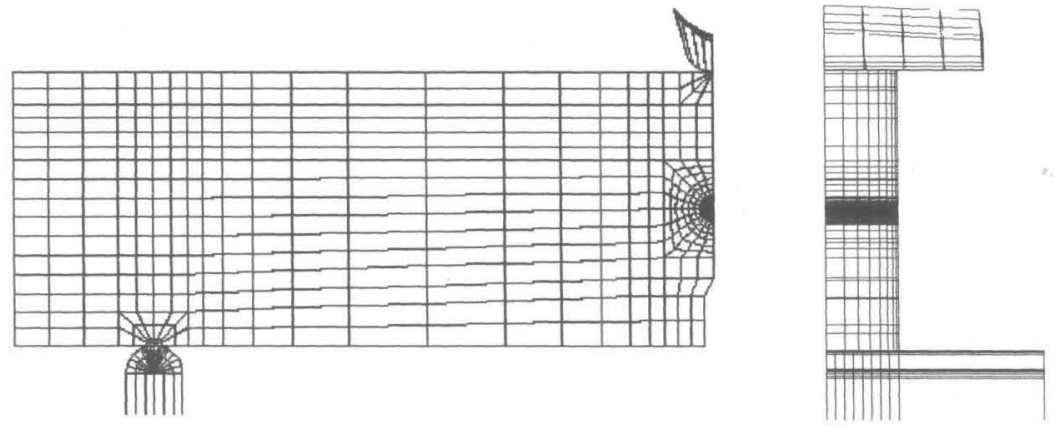

Figure 3: Finite element mesh, case $B=10 \mathrm{~mm}, a / w=0.5$.

Linear elastic behaviour was assumed for the projectile, bar and support material with the following properties: Young modulus $E=200 \mathrm{GPa}$ and Poisson coefficient $\nu=0.3$. The mass density of this material was $\rho=7850 \mathrm{~kg} / \mathrm{m}^{3}$.

For the specimen material (7075-T651 aluminum alloy) an elastic-viscoplastic behaviour with Young modulus $E=72 G P a$ and Poisson ratio $\nu=0.3$ was considered. The viscoplastic constitutive law used was:

$$
\bar{\sigma}=\sigma_{0}\left(\varepsilon^{p l}\right) \cdot\left(1+\frac{\dot{\varepsilon}^{p l}}{D}\right)^{\frac{1}{n}}
$$

where $\varepsilon^{p l}$ is the equivalent plastic strain and $\dot{\varepsilon}^{p l}$ the equivalent plastic strain rate. The dependence of $\sigma_{0}$ on $\varepsilon^{p l}$ was computed as $\sigma_{0}\left(\varepsilon^{p l}\right)=300 \cdot \varepsilon^{p l}+550\left(\sigma_{0}\right.$ expressed in $\left.M P a\right)$. The values of the 
parameters $D$ and $n$ were: $D=9164$ and $n=0.93$. The mass density of this material was $\rho=2800$ $\mathrm{kg} / \mathrm{m}^{3}$. The dynamic cases solved correspond to a projectile impact velocity of $10 \mathrm{~m} / \mathrm{s}$.

The $S I F$ can be obtained from the general path independent dynamic J integral, $J^{\prime}$, derived by Nishioka and Atluri [17](see also [18]). For a mode I stationary crack (as in our case), the $S I F$ of the crack in the plane of symmetry, $\left.K_{I}(t)\right|_{s p}$, was calculated, as in this section purely plane strain conditions exist, as:

$$
\left.K_{I}(t)\right|_{s p}=\sqrt{\frac{E J_{1}^{\prime}(t)}{1-\nu^{2}}}
$$
plane.

where the dynamic $\mathrm{J}$ integral was computed along integration paths contained in the symmetry

For comparison, static analysis was performed by applying an imposed displacement at nodes of the specimen in contact with the input bar, obtaining the SIF from the conventional J integral.

\section{RESULTS}

Figure 4 shows the evolution of $\left.K_{I}\right|_{s p}$ versus $C M O D$ measured at the points on the surface of the specimen, $\left.C M O D\right|_{s f}$, for the case of a dynamic test on a specimen of $B=10 \mathrm{~mm}$ and a ratio $a / W$ of 0.5 .

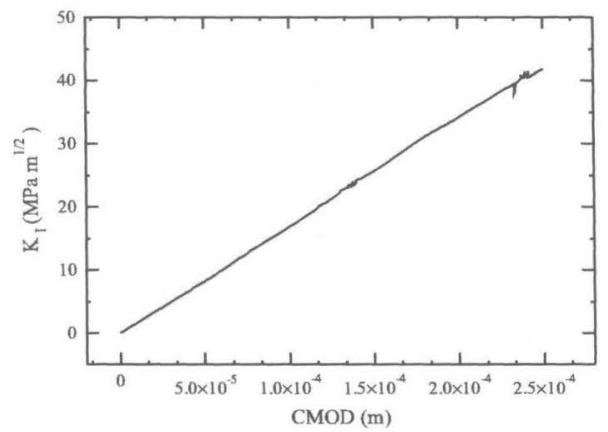

Figure 4: Relationship between $\left.K_{I}\right|_{s p}$ and $\left.C M O D\right|_{s f}$.

A linear relation can be drawn between $S I F$ and $C M O D$, i.e:

$$
\left.K_{I}(t)\right|_{s p}=\left.k_{3 D} \cdot C M O D(t)\right|_{s f}
$$

This result was obtained for all the cases analyzed.

In bidimensional situations the $S I F$ can be related to the $C M O D$ as:

$$
K_{I}(t)=k_{2 D} \cdot C M O D(t)
$$

where the constant $k_{2 D}$ is [19]:

$$
k_{2 D}=\frac{E \sqrt{W}}{4 a} \frac{k_{\beta}(\alpha)}{v_{\beta}(\alpha)}
$$

In this expression, $k_{\beta}(\alpha)$ and $v_{\beta}(\alpha)$ are non-dimensional functions depending on $\alpha(\alpha=a / w)$ and $\beta(\beta=L / W)$ ratios, and their expressions are found in Guinea el al. [19]. For the case $\beta=4$, they are: 


$$
\begin{gathered}
k_{\beta}(\alpha)=\frac{\sqrt{\alpha}}{(1-\alpha)^{\frac{3}{2}}(1+3 \alpha)}\left(1.9+0.41 \alpha+0.51 \alpha^{2}-0.17 \alpha^{3}\right) \\
v_{\beta}(\alpha)=0.76-2.28 \alpha+3.87 \alpha^{2}-2.04 \alpha^{3}+\frac{0.66}{(1-\alpha)^{2}}
\end{gathered}
$$

To determine three-dimensional effects in the $S I F-C M O D$ relationship, the ratio between the slopes $k_{3 D}$ and $k_{2 D}$ were obtained and plotted in figure 5(a) (dynamic cases) and 5(b) (static cases).

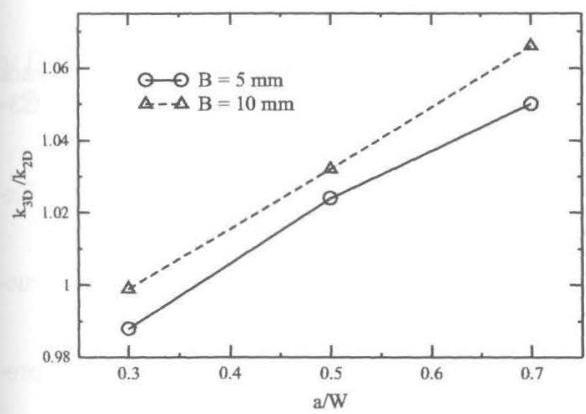

(a) Dynamic cascs.

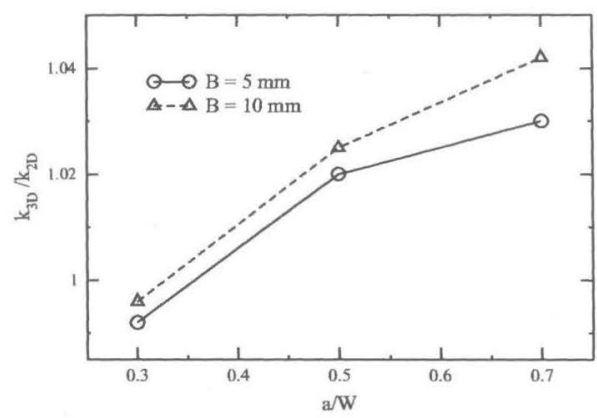

(b) Static cases.

Figure 5: Ratios of $k_{3 D}$ and $k_{2 D}$ slopes.

These figures show that in all the cases considered, except for $a / W=0.3$, the slope calculated from the $3 D$ analysis is greater than the $2 D$ plane strain slope, specially for thin specimens $(B=5$ $\mathrm{mm})$. This indicates that the use of $2 D$ plane strain solutions can slightly underestimate the values of dynamic fracture initiation toughness for specimens with ratio $a / W$ above 0.4 .

\section{CONCLUDING REMARKS}

A three-dimensional numerical simulation of the impact bending fracture test was performed by the Finite Element Method. The model includes the projectile, the input bar, the specimen and its supporting device. The relationship between the Stress Intensity Factor and the Crack Mouth Opening Displacement was examined and compared with that of the bidimensional one. It was concluded that the slope of the linear relationship between the SIF and CMOD calculated from the $3 D$ numerical analysis is greater than that with $2 D$ plane strain solutions, normally used to interpret the fracture test results, so, the $K_{I d}$ determined from $C M O D$ measurement and $2 D$ plane strain relations may be slightly underestimated in specimens with ratio $a / W$ above 0.4 .

\section{Acknowledgements}

This work was supported by the Comisión Interministerial de Ciencia y Tecnología under Grant PB98-0027.

\section{References}

[1] C. Ruiz, R. Mines, The Hopkinson Pressure Bar: An alternative to the instrumented pendulum for Charpy test, International Journal of Fracture 29 (2) (1985) 101-109.

[2] T. Yokoyama, Determination of dynamic fracture-initiation toughness using a novel impact bend test procedure, Journal of Pressure Vessel Technology 115 (2) (1993) 389-397. 
[3] C. Bacon, J. Färm, J. Lataillade, Dynamic fracture toughness determined from load-point displacement, Experimental Mechanics 20 (1) (1994) 217-223.

[4] L. Rubio, Determinación de parámetros de fractura dinámica a alta velocidad de deformación, Ph.D. thesis, Carlos III University of Madrid (1999).

[5] J. Beinert, J. Kalthoff, Experimental determination of dynamic stress intensity factors by shadow patterns, in: G. Sih (Ed.), Mechanics of Fracture, Vol. 7, La Hague: Martinus Nijhoff, 1981, pp. 281-330.

[6] K. Ravi-Chandar, W. Knauss, An experimental investigation into dynamic fracture: I. Crack initiation and arrest, International Journal of Fracture 25 (1984) 247-262.

[7] F. Benitez, L. Andrade, In-plane impact loading of composites: Optical evaluation and crack severity assessment for Graphite-Epoxy, Journal de Physique IV France 7 (1997) C3-169-C3176.

[8] J. Dally, D. Barker, Dynamic measurements of initiation toughness at high loading rates, Experiemental Mechanics 28 (3) (1988) 298-303.

[9] B. Crouch, Finite element modeling of the three-point bend impact test, Computers and Structures 48 (4) (1993) 167-173.

[10] K. Kishimoto, S. Aoki, M. Sakata, Simple formula for dynamic stress intensity factor of precracked Charpy specimen, Engineering Fracture Mechanics 13 (3) (1980) 501-508.

[11] K. Kisihimoto, K. Kuroda, S. Aoki, M. Sakata, Simple formulae for dynamic fracture mechanics parameters of elastic and viscoelastic three point bend specimens based on Timoshenko's beam theory, in: S. Valluri, DMR Taplin, P Rama-Rao, JF Knott and R Dubey (Eds.), Proceeding of 6th International Conference on Fracture. New Delhi (India), Vol. 5, Pergamon, Oxford, 1984, pp. 3177-3184.

[12] L. Rubio, J. Fernández-Sáez, C. Navarro, Determination of dynamic fracture-initiation toughness using three-point bending tests in a modified hopkinson pressure bar, Experimental Mechanics 43 (2) (2003) In press.

[13] C. Popelar, C. Anderson, Jr, A. Nagy, An experimental method for determining dynamic fracture toughness, Experimental Mechanics 40 (4) (2000) 401-407.

[14] A. Rosakis, K. Ravi-Chandar, On the crack tip stress state: An experimental evaluation of three-dimensional effects, International Journal of Solids and Structures 22 (2) (1986) 121-134.

[15] R. Narashiman, A. Rosakis, Three-dimensional effects near a crack tip in a ductile three point bend specimen, Part I: Numerical investigations, Journal of Applied Mechanics (2) (1990) $121-134$.

[16] ABAQUS User' s Manual Version 6.3, Hibbitt, Karlsson \& Sorensen, Inc, 2002.

[17] T. Nishioka, S. Atluri, Path independent integrals, energy release rates, and general solutions of near-tip fields in mixed-mode dynamic fracture mechanics, Engineering Fracture Mechanics 18 (1983) 1-22.

[18] T. Nishioka, On the dynamic J Integral in dynamic fracture mechanics, in: G. P. Cherepanov (Ed.), FRACTURE: A Topical Encyclopedia of Current Knowledge Dedicated to Alan Arnold Griffith, Krieger Publishing Company, Malabar, Florida, USA, 1998, pp. 575-617.

[19] G. Guinea, J. Pastor, J. Planas, M. Elices, Stress intensity factor compliance and CMOD for a general three-point bend beam, International Journal of Fracture 89 (3) (1998) 103-116. 